\title{
Resonant Mode Detection for Fast Determination of Structural and Material Parameters of MEMS
}

\author{
Meinig, Marco ${ }^{\mathrm{a}}$; Kurth, Steffen ${ }^{\mathrm{a}}$; Shaporin, Alexey ${ }^{\mathrm{b}}$; Bauer, Wilfried ${ }^{\mathrm{c}}$; Gessner, Thomas ${ }^{\mathrm{a}, \mathrm{b}}$ \\ ${ }^{a}$ Fraunhofer ENAS, Reichenhainer Str. 88, 09126 Chemnitz, Germany \\ ${ }^{\mathrm{b}}$ Chemnitz University of Technology, Reichenhainer Str. 70, 09126 Chemnitz, Germany \\ ${ }^{c}$ Polytec GmbH, Polytec-Platz 1-7, 76337 Waldbronn, Germany
}

\begin{abstract}
Fast and nondestructive determination of structural and material parameters of mass-spring-damper systems is a key issue in wafer level MEMS test. The knowledge of these parameters allows the selection of defective devices before the cost intensive packaging step and the control of the fabrication process. The proposed method addresses this problem with a non-contact measurement technique based on laser Doppler interferometry. Measured frequency response functions and natural frequencies obtained from finite element simulations are used to calculate structural dimensions and material properties of the device under test. On the example of MEMS scanner arrays for Hadamard transform optics, the method is demonstrated and results are validated by scanning electron microscopy. A mean error of $3 \%$ between the two measurement techniques was observed, which is very promising considering a measurement duration of approximately 4 seconds per device. Techniques for efficient excitation of micromechanical structures in a broad frequency range are presented, as well as algorithms for the precise determination of natural frequencies and the detection of mode interchange.
\end{abstract}

\section{Introduction}

The fabrication of microelectromechanical systems (MEMS) is subject to inherent variations. Deviations are introduced during the generation of masks, through lithography, etching and bonding processes. Critical parameters are thickness and width of elastic beams and mechanical stress in the system. The deviations lead to a certain amount of devices per wafer, which are outside of the specifications. If not detected at wafer level, they are further processed and finally packaged. Considering the overall costs of a device, the packaging step is one of the most expensive. It contributes to roughly $50 \%$ of the costs. Expenses can be saved by identifying defective devices as early as possible in the fabrication process. Therefore the determination of structural and material parameters at wafer level is from greatest interest in MEMS mass fabrication. Furthermore the knowledge of these parameters gives detailed information about the process, allows process control, adaption and the increase of the fabrication yield.

Techniques generally used for determining structural dimensions and material properties in research and development are scanning electron microscopy (SEM), atomic force microscopy (AFM), Raman spectroscopy and Xray diffraction (XRD). For mass fabrication, where time and costs are the limiting factors, they are not suitable. Promising new methods combine theoretical analysis of the device under test with measurement results. They are based on dynamic properties, for which fast and precise measurement techniques, such as laser Doppler interferometry, are available.

For simple structures, an analytical model can be derived. Such a model allows the direct calculation of structural dimensions from measured resonant frequencies [1]. Properties of complex structures, as well as material characteristics, can be determined with the parameter identification method [2]. It combines measured natural frequencies with results from a parametric finite element (FE) simulation. The method was adapted for pressure sensors [3] and micromirror scanners [4], but was not validated by comparative measurements.

In this contribution efficient methods for the excitation of micromechanical structures are investigated. These include the generation of broadband high voltage signals and the excitation with fringing electric fields. Special electrodes of indium tin oxide (ITO) are developed. The vibration modes and natural frequencies are estimated with techniques developed in modal analysis. Different algorithms are investigated. The parameter identification method is finally tested on MEMS scanner arrays and validated by SEM measurements.

\section{General approach to structural and material parameter determination}

There are several precise methods available for determining structural and material parameters of MEMS massspring-damper systems. Scanning electron microscopy can measure structural dimensions down to $1 \mathrm{~nm}$. High resolution images of the surface are generated by scanning a high-energy electron beam above the structure. The reactions of the electrons with the device under test (elastic, inelastic scattering and electromagnetic radiation) are measured by different detectors and images are calculated. The structural dimensions are determined from the images. Attention has to be given to the adjustment of the structure in the SEM, because misalignment leads to falsification of dimensions in the image and measurement errors. X-ray diffraction is a method for the determination of physical properties and chemical compositions of materials. The scattered intensity of an X-ray beam is measured as a function of different parameters. The method needs thorough and time consuming preparation of the samples. Raman spectroscopy is used to characterize mechanical stress and strain in materials. It is a light scattering technique, where radiations of different wavelengths are produced through inelastic scattering. The technique is fast and nondestructive, but not accurate enough for the detection of light stress occurring in microstructures. All of these methods are very precise and widely used in research and development. For measurements at wafer level in the 
production line, they are not suitable. Some methods need a careful preparation of the samples and are generally time consuming, others do not provide the demanded resolution. In mass production time and costs are the critical factors. Methods are needed which are fast, nondestructive and suitable for the cleanroom environment.

The dynamic motion and velocity of a structure in out of plane direction can efficiently be measured with laser Doppler interferometers (LDI). The velocity is obtained from the frequency or phase difference of a split laser beam, where one beam is reflected on a reference mirror and the other on the device under test. The motion of the structure modulates the wavelength of the measurement beam through Doppler shift. The two beams are collected and interfered on a photodetector. A frequency spectrum of vibration up to several $\mathrm{MHz}$ can be measured in less than one second. Through excitation of the structures with a defined broadband signal and simultaneous response measurements, frequency response functions (FRF) can be calculated by numerically dividing the output spectrum through the input spectrum. Reduction of random noise is possible by averaging the complex signals and calculating the FRF with the so called $\mathrm{H}_{1} / \mathrm{H}_{2}$ method. The characteristic of the FRF is determined through the structural dimensions and material properties of the microstructure. Generally mechanical structures can be modeled as massspring-damper systems and described through the equation of motion. Their dynamical properties, such as natural frequencies, modal damping and mode shapes of vibration are determined through their structural dimensions and material properties. Dynamic properties can be calculated analytically or numerically from a model of the system by solving the eigenproblem of the system matrices or using FE methods. They can also be determined from measured frequency response functions of fabricated structures, with curve fitting techniques developed in modal analysis. By combining natural frequencies obtained from measured frequency response functions with those from FE simulations, structural dimensions and material properties of the structure under test can be estimated. The parameter identification method is displayed schematically in Fig. 1.

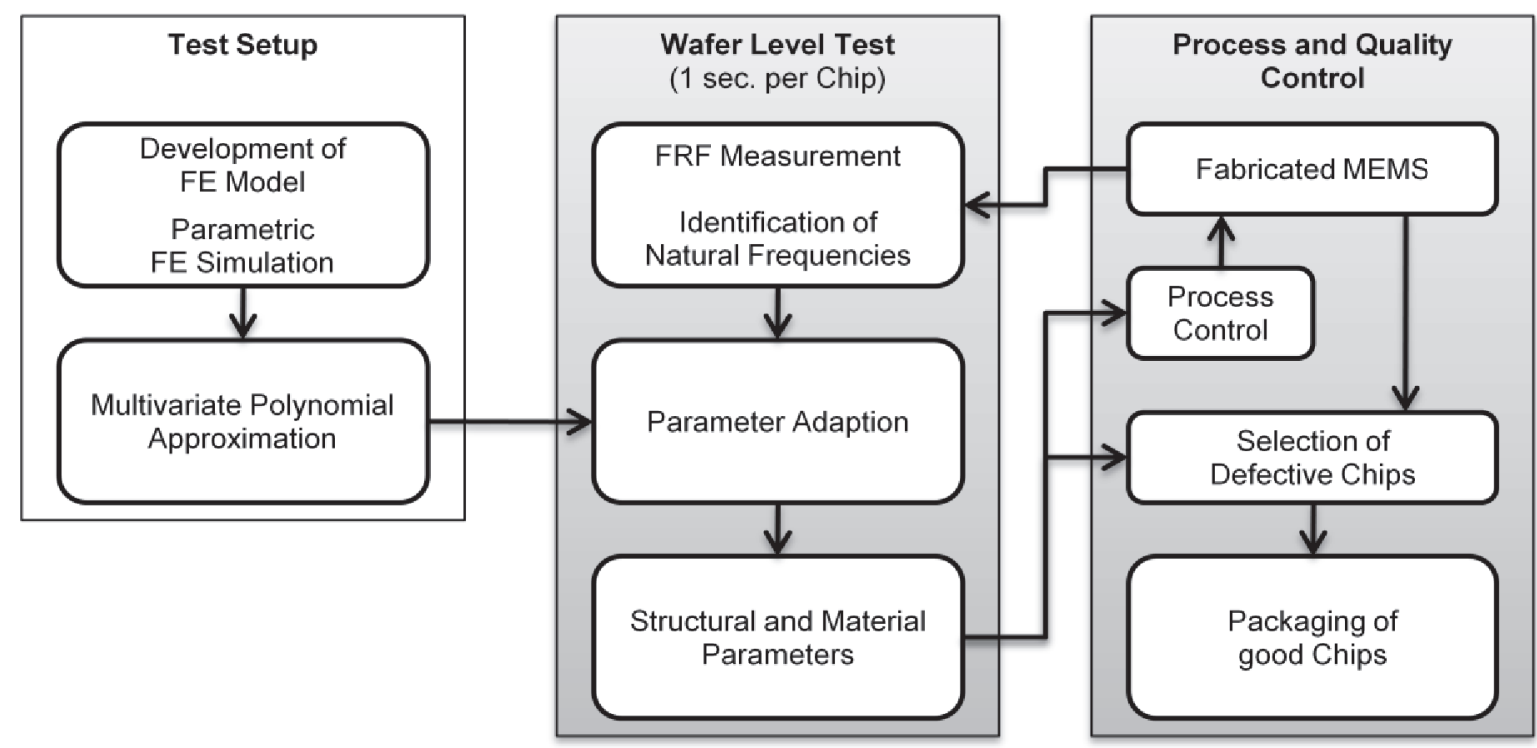

Fig.1: Schematic of parameter identification method with integration into MEMS fabrication

At the test setup a model of the microstructure is developed. In a theoretical modal analysis natural frequencies and mode shapes of vibration are calculated from the model. The simulation must be done parametrically, varying the properties which should later be determined from measurements. The parameter range depends on the possible process variations. The simulation model must be generated thoroughly, implementing every detail contributing to the modal parameters. Afterwards the results are fitted to polynomials. The degree of the polynomials depends on the type of structure and has to be evaluated by calculating the mean error of the approximation. The sensitivity of each calculated natural frequency to the parameters must be calculated. This can be done with the Jacobian matrix. For the identification method, these natural frequencies have to be chosen, which are most sensitive to the interesting structural and material parameters and which can be measured easily with the LDI. The described steps are time consuming and involve user interaction, but they have to be done only ones for every type of structure at the test setup.

During wafer level MEMS test only the FRFs are measured. The FRF must contain all chosen natural frequencies. The measurement point for the LDI has to be chosen carefully. Natural frequencies are estimated from the FRF with the help of modal analysis techniques. In an optimization algorithm based on the least squares method, the polynomials are adapted to the measured natural frequencies. The structural and material parameters are the unknown variables. The least squares problem can be solved with the Levenberg-Marquardt algorithm. At the end of the optimization process the parameters are determined and can be used for process and quality control, or the selection of defective devices. 


\section{Single aspects of MEMS measurement}

\subsection{Excitation of mass-spring-damper systems}

Sufficient excitation of the device under test is an important issue in response measurement. The bandwidth of excitation must be equal to the bandwidth of the calculated FRF. In modal testing and vibration analysis excitation forces are commonly generated with electrodynamic shakers or impulse hammers. For wafer level test of MEMS these methods are not generally suitable. In comparison to macroscopic mechanical systems, MEMS can have natural frequencies in the $\mathrm{MHz}$ range. Examples are high pressure sensors, with very stiff membranes. Shakers provide broadband excitation in the lower $\mathrm{kHz}$ range and cannot excite the higher modes. Impulse excitation also has major drawbacks. The impulse must be short and with large amplitude to stimulate high frequencies. Such impulses or shocks can drive the micromechanical system in the nonlinear range or damage it duo to overload.

Structures can also be excited with electrostatic forces. The principle is commonly used as driving or detection mechanism in MEMS. Micromirrors for example are deflected by built-in electrodes located under the mirror plate. The strength of the field and the resulting force $F_{e l}$ depend on the voltage $V$, the gap $d$ and the area $A$ of the electrodes, using a simple approximation for parallel plates (Eq. 1). The variable $x$ denotes the deflection of the movable structure.

$$
F_{e l}=\frac{V^{2}}{2} \frac{\varepsilon A}{(d-x)^{2}}
$$

At wafer level, where the devices are not fully assembled, built-in electrodes are not available. An external electrode, placed above the structures surface, has to be used instead. Different electrode shapes such as needles or plates of metal are possible. Especially suitable for the parameter test are fringing field electrodes made of indium tin oxide on a glass substrate (Fig. 2). They are transparent for the laser of the LDI and optical inspection through a microscope.

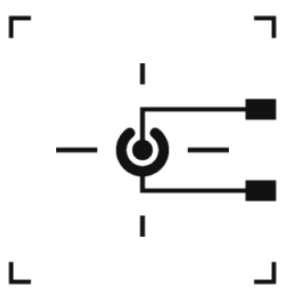

(a)

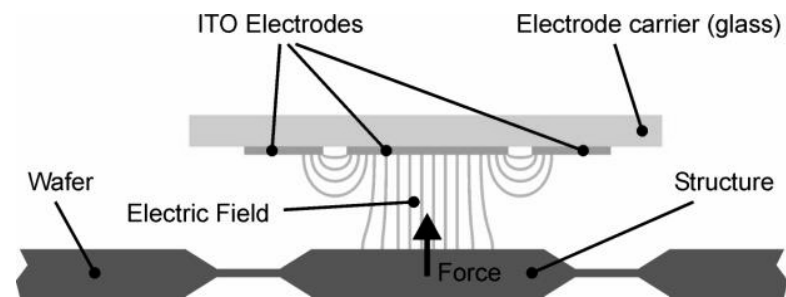

(b)

Fig. 2: (a) Schematic of concentric round excitation electrode, (b) Cross-sectional view of concentric round electrode above a microstructure

The electrodes can be efficiently fabricated with microelectronic processes. Built in a probe card of standard size, they fit in an automatic wafer prober. The distance between the electrode and the wafers surface is measured with laser triangulation, also built in the probe card. Normally distances from $50 \mu \mathrm{m}$ to $100 \mu \mathrm{m}$ are used. ITO electrodes can be operated up to $400 \mathrm{~V}$ and provide strong fringing electric fields in comparison to other electrode shapes. Attention has to be given to two effects of electrostatic excitation of mass-spring-damper systems.

- Electrostatic excitation of MEMS is generally connected with electrostatic softening of the mass-springdamper system. The electrostatic force acts in opposite direction to the mechanical spring force. The stiffness of the whole system $k_{\text {sum }}$ is reduced by the electrostatic stiffness (Eq. 2). Electrostatic softening can lead to a system with significant lower natural frequencies.

$$
k_{\text {sum }}=k_{m e c h}-V^{2} \frac{\varepsilon A}{(d-w)^{3}}
$$

In MEMS parameter test the influence is usually negligible duo to small deflections, but must be checked at the setup of the test.

- $\quad$ The voltage amplitude influences the electric field to the power of two (Eq. 1). Applying a sine signal with a DC offset, the frequency spectrum contains also a component at two times the frequency of the sine signal. Usually this has to be considered, while interpreting the calculated frequency response function. If only natural frequencies have to be determined, the effect does not influence the results.

\subsection{Generation of broadband excitation signals}

The determination of frequency response functions is achieved time efficient through broadband excitation of the micromechanical structures and simultaneous response measurements. Suitable waveforms with a broadband frequency spectrum are multisine signals (Eq. 3).

$$
u(t)=\sum_{k=1}^{N} A_{k} \sin \left(2 \pi f_{k} t+\varphi_{k}\right)
$$


Multisine signals are the sum of a set of sine waves and can be generated with an arbitrary amplitude $A_{k}$ and phase $\varphi_{k}$ spectrum. Broadband excitation signals usually have a constant amplitude spectrum. Special attention has to be given to the phase spectrum. If it is constant, the time domain signal will show the characteristic of an impulse function. Such impulses are not preferable. They can lead to overload, clipping or nonlinear excitation of the structure. In vibration analysis this peaks are described as impact. A measure is the crest factor CF. It is defined as the ratio of a signals peak value to its RMS value (Eq. 4).

$$
C F(u)=\frac{\|u\|_{\infty}}{\sqrt{\frac{1}{N} \sum_{i=1}^{N} u_{i}^{2}}}
$$

Low crest factors can be reached through either a random phase spectrum (Fig. 3a) or a mathematically optimized phase spectrum (Eq. 5, Fig. 3b) [5, 6].

$$
\varphi_{k}=\pi \cdot \frac{(k-1)^{2}}{N}, k=1 \ldots N
$$

The crest factor of a signal with random phase spectrum is usually larger as of that with a phase spectrum according to Eq. 5. It can be improved by generating a large number of random phase spectra, afterwards calculating the crest factor of each resulting multisine signal and choosing the signal with the lowest crest factor.

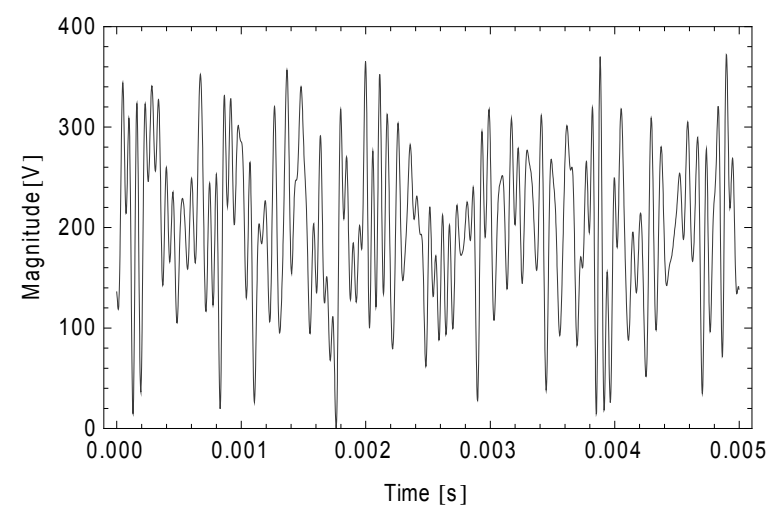

(a)

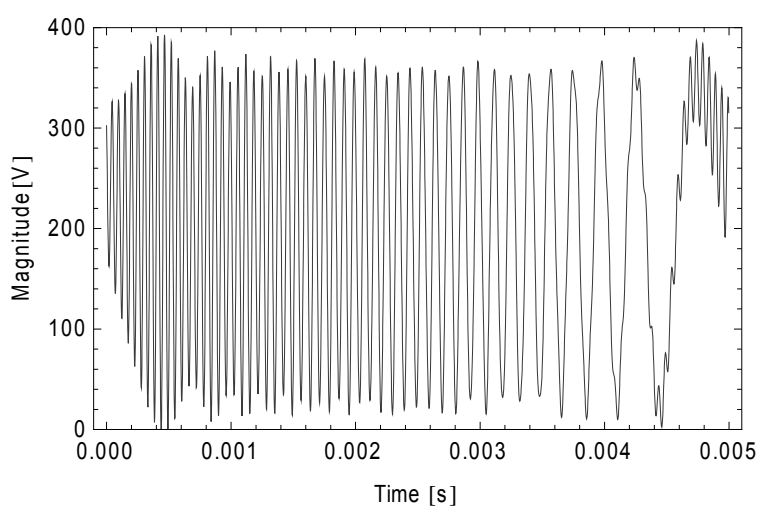

(b)

Fig. 3: Multisine signals with (a) Random phase spectrum $C F=2.3$,

(b) Newman phase spectrum $C F=1.6$

In general it is not necessary to excite the full frequency range of the frequency response function with a constant amplitude spectrum. In most cases only natural frequencies have to be determined, with as much accuracy as possible. A high signal to noise ratio (SNR) and coherence close to one is preferable. The location and deviation of the natural frequencies are known from the parametric FE simulation. Exciting only the frequency ranges around natural frequencies, distributes the whole power of the high voltage amplifier in these ranges. The coherence, which is a measure for the statistic certainty of each data point, can be significantly increased (Fig. 4).

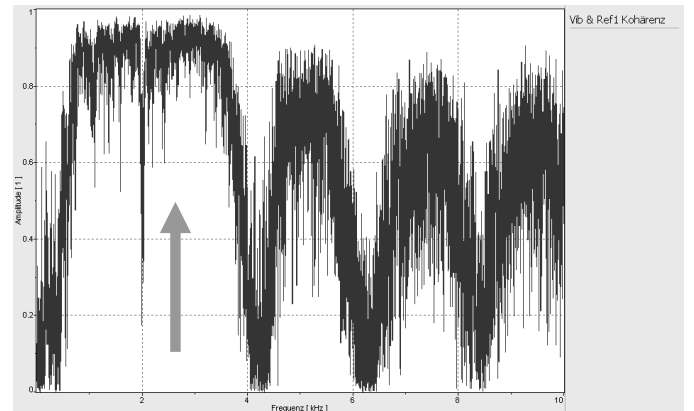

(a)

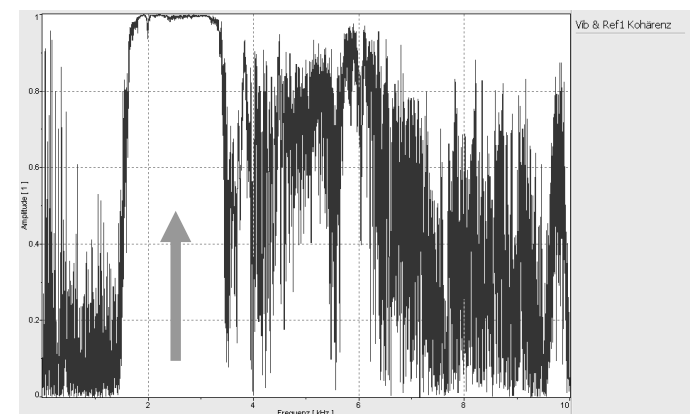

(b)

Fig. 4: Coherence function obtained through (a) Excitation of entire spectral range,

(b) Excitation of selected vibration mode

The amplitude spectrum of a multisine signal can be generated with maximum amplitude around the natural frequencies and zero amplitude between them. 


\subsection{Determination of natural frequencies}

A FRF measurement results in a set of complex valued data from which the dynamic properties of the mass-springdamper system can be determined. The estimation methods are mainly developed in the field of modal analysis. Four different methods have been analyzed for the use with the parameter identification method. The simplest method is the peak picking method. The natural frequency is obtained from the peak in the mobility function (Tab. 1a), which occurs exactly at that frequency. Mobility is a FRF, where the velocity response is divided by the excitation force. The method uses only one data value for the analysis. It is sensitive to noise and the frequency resolution of the mobility function. The method can be improved by fitting a model function to the data values surrounding the natural frequency. The Lorentzian function which is commonly used for fitting resonance peaks is suitable. It can be improved with additional linear and quadratic correction terms. This method gives good results also on data with a low signal to noise ratio (Tab. 1b). A more sophisticated modal analysis technique which can determine the natural frequencies exact using the complex data format is the circle fitting method. The mobility function describes a characteristic circle in the surrounding of the natural frequency. An analytic circle [7] can be fit to the data and the natural frequency is obtained from the value where the sweep rate of the frequency around the circle reaches its maximum (Tab. 1c). The method provides exact results, but needs data with a high signal to noise ratio. The rational fraction polynomial [8] method uses complex data as well. It is a MDoF method and can calculate all modes in a specified frequency range in one step. The method can also be used to fit a single natural frequency from a mobility function (Tab. 1d).

Tab. 1: Comparison of identification algorithms

\begin{tabular}{|c|c|c|c|c|}
\hline (a) Peak Picking Method & (b) Enhanced Lorentzian Fit & (c) Circle Fit & (d) Rational Fraction \\
Polynomial Method
\end{tabular}

If a structure has vibration modes with close natural frequencies and structural dimensions as well as material properties which can vary in a large range, it can happen, that vibration modes interchange. The vibration modes and the corresponding parameters are known beforehand from the parametric FE simulation. By measuring the FRF of two or more locations of a structure, also phase information between the locations can be obtained. Choosing the right measurement locations, this information is unique for each vibration mode and can additionally be compared with simulation results.

\section{$4 \quad$ Results and discussion}

The parameter identification method was tested on MEMS scanner arrays (Fig. 5a). The scanners are made of silicon using dry and wet etching processes. They consist of a rectangular mirror plate with elastic beams on one of their long sides. The mirrors are bonded on an electrode carrier with aluminum electrodes.

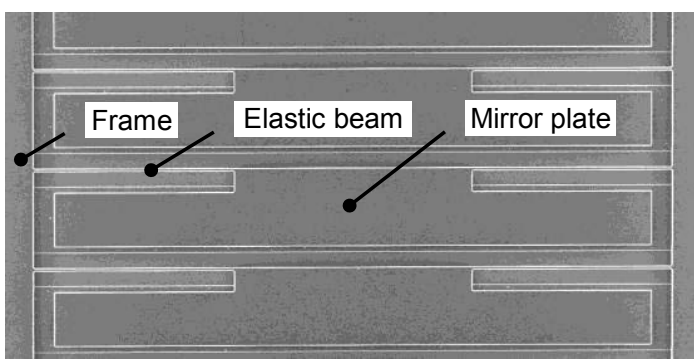

(a)

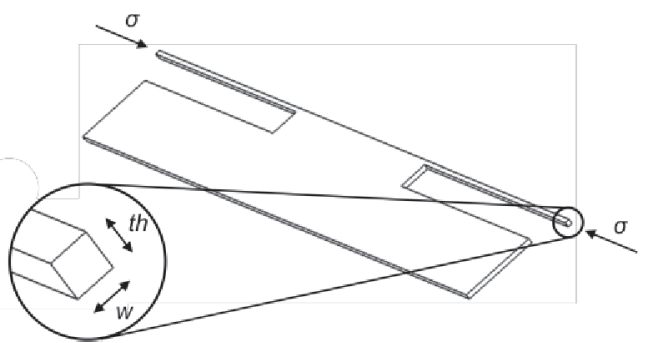

(b)

Fig. 5: (a) SEM image of MEMS scanner array, (b) Schematic of single microscanner 
The dynamic properties of the scanners are determined through the thickness th and width $w$ of the elastic beams and the mechanical stress $\sigma$ in the structure (Fig. 5b). The thickness and width are determined during the mask layout, lithography and the etching process. They vary slightly for every mirror in an array. Depending on the parameters of the wafer, they also vary from array to array. Mechanical stress is introduced in the system through the bonding process.

At the test setup a parametric FE simulation was made, varying the parameter thickness and width in a range from $3 \mu \mathrm{m}$ to $6 \mu \mathrm{m}$ in steps of $0.5 \mu \mathrm{m}$ and the mechanical stress from $-15 \mathrm{MPa}$ to $15 \mathrm{MPa}$ in steps of $5 \mathrm{MPa}$. The first six natural frequencies and mode shapes are calculated for each combination of the parameters. The first, third, fifth and sixth mode was chosen for the identification method. For each mode a polynomial was fitted to the chosen natural frequencies to obtain them as a function of thickness, width and mechanical stress. The natural frequencies of the micromirror scanners where estimated from FRFs measured through electrostatic excitation. A bandwidth of $100 \mathrm{kHz}$ was used and excited with a broadband high voltage signal of $400 \mathrm{~V}$ amplitude. The polynomials are adapted to the measured natural frequencies and thickness, width and mechanical stress are calculated.

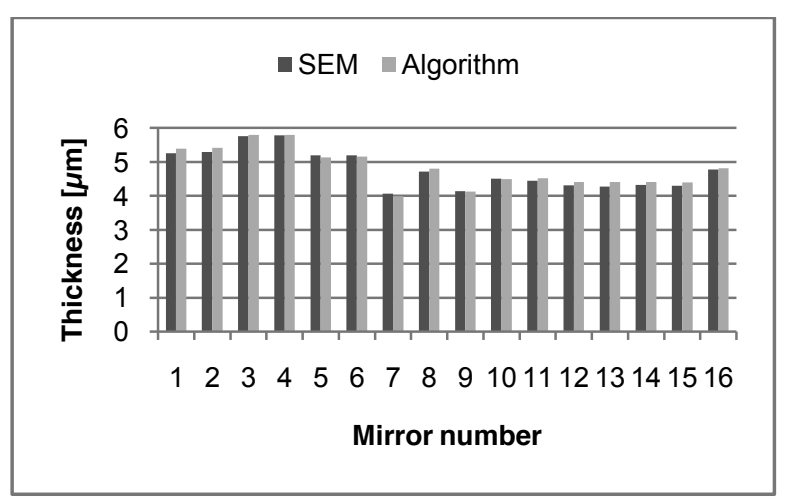

(a)

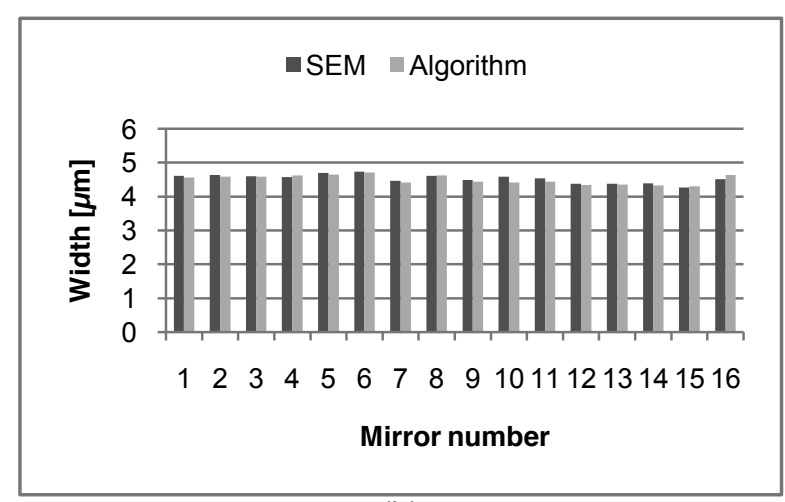

(b)

Fig. 6: Comparison of identification results (a) Thickness of elastic beams, (b) Width of elastic beams

Single mirrors from different locations of a scanner array and from different arrays of a wafer are extracted and measured with scanning electron microscopy. A comparison of the results shows an average error of $3 \%$ between the two measurement methods (Fig. 6).

\section{Conclusion}

With the parameter identification method structural dimensions and material properties of micromechanical massspring-damper systems can be determined precise and nondestructive at wafer level. The method is fast in comparison to existing technologies and suitable for the cleanroom environment. From the determined parameters process influencing variables such as duration of etching can be estimated, which makes it possible to control the process more precise. In comparison to existing measurement technologies different parameters can be obtained during a single measurement. An average identification procedure is completed in 4 seconds.

\section{$6 \quad$ Literature}

[1] D.M. Tanner, A.C. Owen, Jr., F. Rodriguez, Resonant frequency method for monitoring MEMS fabrication, Reliability, Testing, and Characterization of MEMS/MOEMS II, 2003, 4980, pp. 220-228

[2] A.V. Shaporin, M. Hanf, W. Doetzel, Novel characterization method for MEMS devices, Proceedings of SPIE, 2005, 5716, pp. 198-206.

[3] S. Michael, S. Kurth, J. Klattenhoff, H. Geissler, S. Hering, Parameter Identification of Pressure Sensors by Static and Dynamic Measurements, DTIP of MEMS \& MOEMS, 2007

[4] S. Kurth, J. Mehner, A. Shaporin, S. Michael, M. Ebert, W. Dötzel, T. Gessner, Determination of dimensional parameters in MEMS components by vibration analyses, Conference Proceedings of IMAC-XXV, Orlando, Florida, Feb. 19-22, 2007, pp. 165ff.

[5] D.J. Newman, An L1 Extremal Problem for Polynomials, Proceedings of the American Mathematical Society, 1965, 16, pp. 1287-1290.

[6] S. Boyd, Multitone Signals with Low Crest Factor, IEEE Transactions on Circuits and Systems, 1986, CAS-33, pp. 1018-1022.

[7] I. Kåsa, A Circle Fitting Procedure and Its Error Analysis, IEEE Transactions on Instrumentation and Measurement, 1976, 25, pp. 8-14

[8] M.H. Richardson, D.L. Formenti, Parameter Estimation from Frequency Response Measurements using Rational Fraction Polynomials, Proceedings of the 1st International Modal Analysis Conference (IMAC I), 1982, pp. 167-181 